\title{
nombalina
}

(8)

\section{II fascismo e le sue storie}

\author{
Autor(es): $\quad$ Bernardi, Alberto De
}

Publicado por: Imprensa da Universidade de Coimbra

URL persistente:

URI:http://hdl.handle.net/10316.2/32185

DOI:

DOl:http://dx.doi.org/10.14195/978-989-26-0810-5_1

Accessed : $\quad$ 26-Apr-2023 15:44:25

A navegação consulta e descarregamento dos títulos inseridos nas Bibliotecas Digitais UC Digitalis, UC Pombalina e UC Impactum, pressupõem a aceitação plena e sem reservas dos Termos e Condições de Uso destas Bibliotecas Digitais, disponíveis em https://digitalis.uc.pt/pt-pt/termos.

Conforme exposto nos referidos Termos e Condições de Uso, o descarregamento de títulos de acesso restrito requer uma licença válida de autorização devendo o utilizador aceder ao(s) documento(s) a partir de um endereço de IP da instituição detentora da supramencionada licença.

Ao utilizador é apenas permitido o descarregamento para uso pessoal, pelo que o emprego do(s) título(s) descarregado(s) para outro fim, designadamente comercial, carece de autorização do respetivo autor ou editor da obra.

Na medida em que todas as obras da UC Digitalis se encontram protegidas pelo Código do Direito de Autor e Direitos Conexos e demais legislação aplicável, toda a cópia, parcial ou total, deste documento, nos casos em que é legalmente admitida, deverá conter ou fazer-se acompanhar por este aviso. 


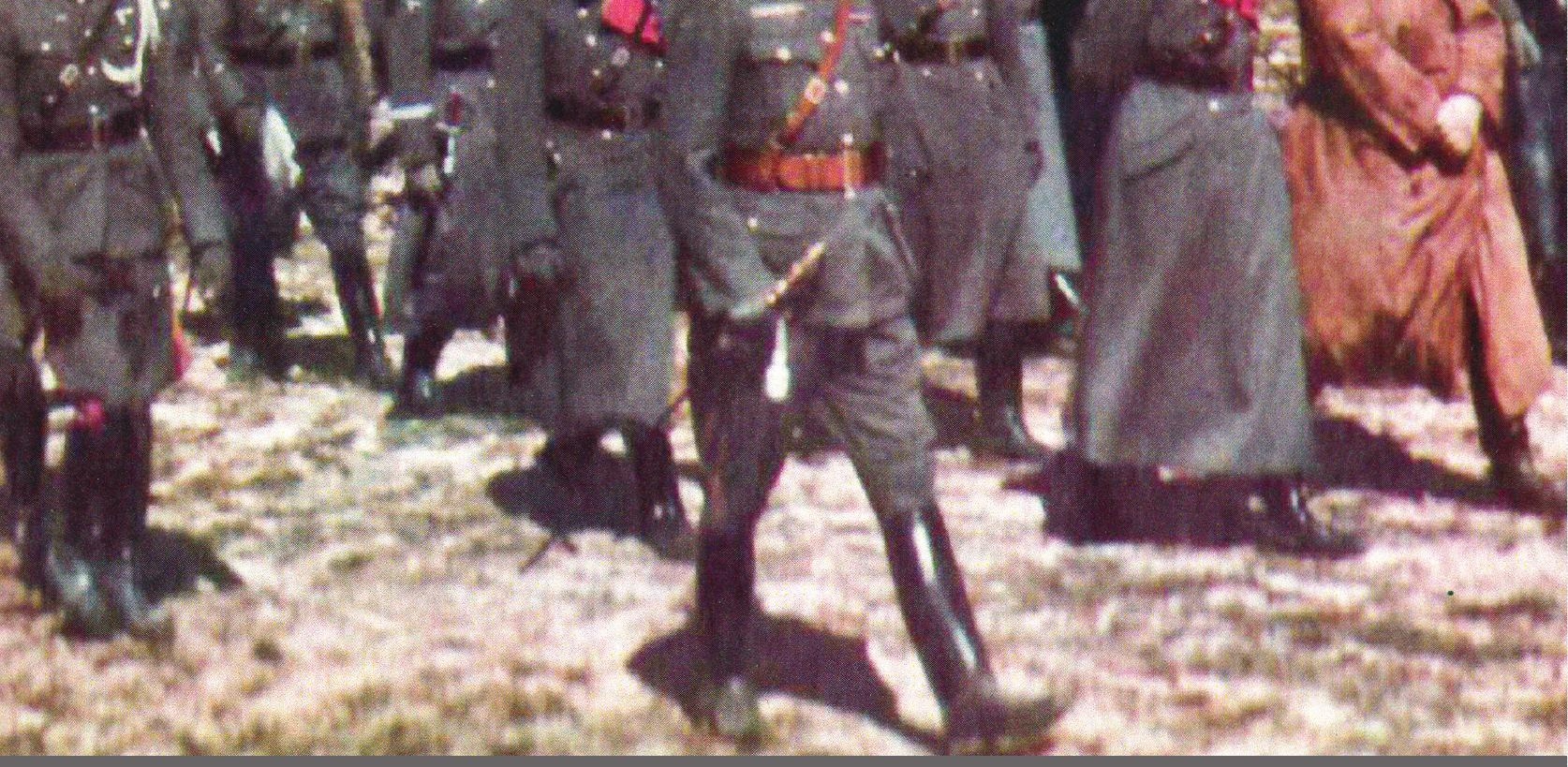

\section{Estados autoritários}

e totalitários

e suas representações

Coordenaação

Luís Reis Torgal

Heloísa Paulo

Coimbra · 2008

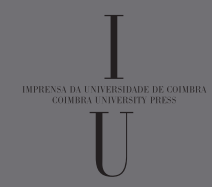




\section{IL FASCISMO E LE SUE STORIE}

Le modalità con cui abbiamo concepito e mantenuto questi nostri incontri, che si sviluppano tra ascolto, proposta e riflessione attorno ad idee e punti di vista eterogenei, ulteriormente arricchiti dalla periodicità con cui le nostre singole comunità scientifiche hanno scelto di incontrarsi e dalla particolare attenzione nei confronti delle diverse generazioni di storici, anche in quest'occasione mi ha permesso di ripensare, riordinare e mettere nuovamente in discussione sotto una nuova luce idee e pensieri su cui ormai da tempo indugio in veste di storico. Così, arricchito e stimolato nuovamente, cercherò anche in questa occasione di dare contributo e seguito a questo nostro progetto collaborativo, che iniziato ormai da qualche tempo rivela ormai chiaramente il suo duplice destino ed obiettivo: da una parte stimolo alla discussione e alla polemica storica, dall'altro e soprattutto occasione per ottimi risultati in termini di apporto alla conoscenza storica.

Disattendendo il proposito iniziale, ossia offrire un breve ed esaustivo inquadramento sulla storia della storiografia fascista, scelgo, grazie agli spunti dei colleghi, un approccio più specifico e comparativo, tenuto conto sia del fatto che l'esperienza del fascismo è "comune" a molti paesi, quantomeno declinata a diverse realtà, e poi del fatto che è esistita in Italia una storiografia sul fascismo fatta da storici fascisti che non è né semplicemente adulatoria, né scientificamente sterile.

Nel processo di costruzione del regime la definizione di una politica culturale capace di stimolare e promuovere un rapporto sempre più stretto tra gli intellettuali e il fascismo ha ricoperto un ruolo tanto particolare quanto ignorato. L'alta cultura non era infatti impermeabile alla penetrazione dell'ideologia fascista, come per lungo tempo ha sostenuto la storiografia antifascista e come hanno smentito ricerche ormai relativamente recenti, e di questo occorre tener conto soprattutto per prendere atto della complessità e della differenziazione che hanno caratterizzato l'insieme delle relazioni tra il mondo della cultura e il regime mussoliniano. Si tratta di un rapporto biunivoco in cui accanto allo sforzo del regime di conquistarsi il consenso degli intellettuali per inglobarli ed utilizzarli nella sua politica di fascistizzazione della società, sta il groviglio difficilmente districabile dei percorsi soggettivi che hanno portato questi studiosi a scegliere il fascismo e a tendere al medesimo fine fascistizzante col loro apporto propagandistico. 
Prese di posizione antifasciste, dunque, non interruppero il flusso di adesioni al fascismo da parte della maggioranza degli intellettuali e degli accademici che proseguì non soltanto perché questi erano tradizionalmente adusi a un rapporto conformista con il potere politico, ma perché erano stati progressivamente persuasi e conquistati dall'autoritarismo nazionalista del fascismo, fino a renderli disponibili a "passare sul corpo della dea libertà" senza eccessivi drammi interiori.

Essi salutarono con unanime entusiasmo la riforma scolastica promossa da Gentile nel 1923, quando assunse la carica di ministro della Pubblica istruzione, così come con fervore parteciparono nel 1925 alla stesura della Enciclopedia Treccani, varata dallo stesso Gentile e ritenuta da Mussolini la più significativa impresa culturale del regime, e sottoscrissero il Manifesto degli intellettuali italiani fascisti agli intellettuali di tutte le nazioni, lanciato nell'aprile dello stesso anno, infine ad accettare di buon grado di svolgere il loro magistero scientifico all'interno delle nuove istituzioni culturali proposte dal fascismo. Non sarebbe altrimenti comprensibile il fatto che, nel novembre 1931, la totalità dei professori universitari - salvo dodici - giurarono fedeltà al regime, quando essa fu loro obbligatoriamente richiesta.

Da questo punto di vista, la vicenda della cultura storica appare emblematica. Tra il 1923 e il 1930 il regime costruì una vasta rete di istituzioni deputate all'organizzazione degli studi storici, sotto l'alta direzione di Gioacchino Volpe che, in questo ambito, svolse la stessa funzione ricoperta in quello più generale e teorico da Giovanni Gentile attraverso l'Istituto fascista di cultura. Nel 1923 venne costituita la Scuola storica nazionale per la raccolta e la pubblicazione delle fonti medievali; nel 1925, per iniziativa diretta dello stesso Volpe, nacque la Scuola di storia moderna e contemporanea, cui seguirono le fondazioni dell'Archivio storico della Svizzera italiana, dell'Archivio storico della Corsica, dell'Archivio storico della Dalmazia, nonché l'istituto di studi romani. L'opera si compì, con la creazione nel 1934 della Giunta degli studi storici, alle cui dipendenze fu posta l'intera rete di istituzioni culturali attive in questo campo disciplinare, e poi con la definitiva integrazione nell'Istituto fascista di cultura di Trino della più prestigiosa rivista storica italiana, la «Rivista storica italiana» fondata nel 1884 da Costanzo Rinaudo), con un intento dichiaratamente militante a favore del nazionalismo e della difesa della "italianità" della cultura storica nazionale.

Possiamo dunque dire che questa storiografia, nel caso italiano, è stata fatta soprattutto da una scuola storica fondata e diretta da Gioacchino Volpe, che a ragione può essere considerato uno dei più grandi storici italiani del Novecento, valutazione peraltro suffragata dal fatto che le sue idee hanno superato i confini nazionali per emigrare all'estero - troviamo i suoi libri anche tra i volumi della biblioteca dell'Università di Coimbra che ci ospita per questo nostro incontro. Uno storico, dunque, di tutto rispetto, la cui interpretazione del fascismo costituisce senza dubbio un punto di riferimento.

Accanto a Gioacchino Volpe, vorrei proporre alla vostra attenzione un'altra figura di rilievo dell'intellettualità fascista, quella del giurista e politico Francesco Ercole, uno storico meno noto ma non meno importante che ha scritto quella che possiamo considerare la prima storia del fascismo, uscita in Italia nel 1936 col titolo molto interessante di La rivoluzione fascista, e ripubblicata nel 1939 in due volumi, con 
il nuovo nome di Storia del fascismo. ${ }^{1}$ Ercole è un personaggio importante anche per il suo "curricula" fascista: iscrittosi nel 1923 al Pnf, fu membro nel 1924 delle commissioni dei Quindici e nel 1925 delle commissioni dei Diciotto per le riforme istituzionali, quindi tra il 1929 e il 1939 deputato, dal 1932 al 1935 membro del Gran consiglio, ma soprattutto fu ministro dell'Educazione nazionale (dal 1932 al 1935) e fondatore della "Giunta di Studi Storici", un organismo di controllo, promozione ed organizzazione degli studi storici in Italia, che esiste ancora oggi.

Partiamo innanzitutto da Gioacchino Volpe. Volpe, nato nel 1876 si è affermato come storico del medievo medievista, come anche il suo antagonista culturale Gaetano Salvemini, una delle figure più importanti dell'antifascismo; quando nel 1922 il fascismo prende il potere, ha più di quarant'anni ed è già un'autorità scientifica assoluta, diversamente da Ercoli che, nato quasi dieci anni dopo di lui, nel 1922 è uno giovane studioso, ai primi passi della carriera universitaria.

Innanzitutto vorrei sottolineare l'operazione politico-culturale che Volpe mette in atto. Volpe coglie che la prima guerra mondiale ha segnato un cambiamento d'epoca storica imponedo agli studi storici di confrontarsi con la contemporaneità, di leggere il presente in chiave storica. Egli intuisce cioè che i processi che si sono messi in moto nell'Italia e nell'Europa tra il 1910 e il 1920 richiedono una loro narrazione, un impegno supplementare di analisi e di indagine da parte degli storici, per fornire all'opinione pubblica sintesi interpretative che entrino nel merito del dibattito politico.

Possiamo quindi dire che Gioacchino Volpe per primo capisce la necessità di un uso pubblico della storia: non di una storia ancella della politica politica, di una agiografia dei vincitori, nel senso di una elaborazione e di una analisi piegata agli interessi del fascismo. Anche se Volpe è un intellettuale fascista, si cimenta con il presente mantenendo sempre la sua fisionomia di storico; Volpe però ritiene che il suo mestiere debba allargarsi e assumere un ruolo civile, oltre che scientifico, diventando strumento della formazione dell'opinione pubblica nell'epoca della società di massa.

In questa veste dunque, egli vira e riorienta tutti gli studi storici italiani con il risultato che per circa quarant'anni, dagli anni Venti fino agli anni Sessanta, la storiografia italiana di dedicò prevalentemente alla storia del Risorgimento e dell'Italia liberale. Gioacchino Volpe fu l'artefice di una generazione di storici che, nati quasi tutti medievisti, diventarono contemporaneisti; e dunque in questo senso Volpe inventa e costruisce la storia contemporanea che sino ad allora in Italia non c'era perché era considerata un "argomento secondario" rispetto al nocciolo duro della riflessione storiografica rappresentato dalla storia del medioevo e dell'età moderna.

Volpe, dunque, ribalta l'opinione che al tempo si nutriva nei confronti dei contemporaneisti; cosicchè mentre prima della lezione di Volpe solo uno studioso del medioevo poteva aspirare a diventare uno storico di vaglia, dunque solo un medievista poteva fare carriera all'Università, dopo l' operazione volpiana la storia contemporanea entra di diritto tra le discipline accademiche. Questo cambiamento di rotta si verifica

${ }^{1}$ Tra gli scritti dell'autore, citiamo: Francesco Ercole, Dal nazionalismo al fascismo: saggi e discorsi, Roma, De Alberti, 1928; La rivoluzione fascista, Palermo, Ciuni, 1936; Storia del Fascismo: vol. 1.: Dai fasci di azione rivoluzionaria alla marcia su Roma, 1915-1922; vol. 2.: Dalla marcia su Roma alla creazione dello stato fascista corporativo e alla proclamazione dell'Impero, 1922-1936, Milano, Verona, A. Mondadori, 1939. 
senza che essa perda la sua forte connotazione politica, legata cioè in maniera esplicita ai suoi usi pubblici, anche in virtù del profilo di intellettuale militante dell'artefice di questo cambiamento che non negherà mai di essere un nazionalista diventato fascista, di essere un intellettuale organico al regime in costruzione, e quindi di orientare inevitabilmente tutta la storia in virtù di questo disegno e progetto di costruzione della egemonia culturale del fascismo.

Questa sintetica premessa ci permette di introdurre chi fosse il grande avversario di Gioacchino Volpe: Benedetto Croce, il maestro per antonomia della storiografia italiana, tra Ottocento e Novecento. Filosofo, storico e politico, Croce esercitò il suo magistero intellettuale lontano dalle aule universitarie, non avendo mai puntato a una cattedra e a un riconoscimento addademico, attraverso le sue pubblicazioni ma soprattutto attraverso la rivista «La critica», pubblicata dal 1903 al 1944, che divenne una delle rare voci dell'opposizione che il fascismo decise di tollerare e sicuramente una delle più grandi riviste intellettuali e culturali europee del primo novecento. ${ }^{2}$

Croce fu il primo a lanciare l'idea che tutta la storia fosse storia contemporanea, nel senso che il punto di vista con cui gli studiosi e gli storici guardano al passato è sempre fondamentalmente segnato dalla condizione contemporanea dello storico. $\mathrm{Na}$ l'operazione del suo allievo Volpe va oltre questa idea, è più complessa e mostra di cogliere più nitidamente le nuove frontiere sulle quali questa intuizione metodologica doveva cimentarsi. Egli infatti non solo riconosce che (tutta) la storia è contemporanea, quindi anche lo studio del medioevo, perché la ricerca è influenzata dalla sensibilità, dalla mentalità, dagli interrogativi del presente, ma soprattutto mette in evidenza che la storia contemporanea é un oggetto storiografico fondamentale e legittimo, la cui trattazione, come tutta la ricerca storica, è influenzata dalle condizioni storiche in cui la ricerca è posta; nel caso specifico nel momento nel quale entra in crisi lo stato liberale e un nuovo regime è in costruzione.

Il progetto politico e culturale di Gioacchino Volpe trova la sua sintesi più compiutata nel volume, L'Italia in cammino, ${ }^{3}$ uscito nel '1927, conservato anche nella Biblioteca dell'Università di Coimbra. Il termine ad quem del volume è il 1915, cioè alla vigilia della prima guerra mondiale, quindi non è una storia del fascismo e soprattutto della sua presa del potere. Si occupa piuttosto delle forze materiali e spirituali che stanno alle origini del fascismo. Gioacchino Volpe in questo lavoro mette in evidenza alcuni punti salienti, a partire dal titolo, che mette in evidenza lo stato di dinamicità e di divenire, "in cammino", nel quale si è trovava l'italia nei cinquanta anni successivi all'unificazione nazionale. Ma verso dove si muove l'Italia postunitaria, quale è la meta di questo "cammino"? Ebbene, secondo l'interpretazione di Gioacchino Volpe la storia d'Italia si dirige inevitabilmente verso il fascismo; per meglio dire la storia dell'Italia liberale, che rappresenta il suo oggetto di studio, ha come suo esito più coerente il regime totalitario.

\footnotetext{
${ }^{2}$ Sottotitolata Rivista di letteratura, storia e filosofia, "La Critica» venne fondata nel 1903 da Benedetto Croce e da lui finanziata fino al 1906, anno in cui subentrò l'editore Laterza. Si mantenne in vita fino al 1944, per poi riprendere le pubblicazioni nel 1945 e concludere la sua parabola nel 1951, con una periodicità più ridotta e con il titolo di «Quaderni della Critica».

${ }^{3}$ Gioacchino Volpe, L'Italia in cammino, Milano, Treves, 1927.
} 
Questa lettura ha un'importanza notevole, perché è sorretta dall'idea tutta politica che la storia dell'Italia contemporanea sia non solo animata da un progressivo avvicinamento al fascismo, ma soprattutto che la storia dell'Italia unita sia una sorta di antefatto del fascismo, che in quest'ottica appare come il risultato implicito dell'Unità nazionale.

Si tratta di un modello interpretativo opposto a quella di Benedetto Croce, che un anno dopo L'Italia in cammino, darà infatti alle stampe la Storia d'Italia dal 1871 al $1915^{4}$ (e cinque anni dopo la Storia d'Europa nel secolo decimonono ${ }^{5}$ ), anche questa una storia dell'Italia liberale ma diversamente e in contrasto a quella di Volpi, scritta per dimostrare esattamente l'opposto, e cioè che il fascismo è la negazione e non l'inveramento del Risorgimento, è una parentesi nel cammino "indefessamente» ${ }^{6}$ progressivo di quell'Italia liberale che aveva trovato il suo momento più alto nel compromesso giolittiano, considerato da Croce un autentico capolavoro politico. $\mathrm{Ne}$ nascerà una polemica molto forte ovviamente condotta da tutta la stampa del regime contro il filosofo abruzzese, che farà di Croce, intellettuale troppo importante e famoso, un esule in patria, completamente isolato.

Il secondo aspetto che è bene tener presente è relativo alla lettura che Volpi propone circa l'Italia liberale. Egli, interrogandosi sulle ragioni del fallimento dell'Italia liberale, individua come origine causale l'incapacità di quest'Italia di realizzare il suo obiettivo principale, ossia la nazionalizzazione delle masse; una critica che Volpi non si limita a muovere alla sola Italia liberale, ma che estende più in generale all'intera Europa liberale. Il processo di nazionalizzazione delle masse, quindi, è sostanzialmente fallito perché lo stato liberale che ha una base troppo ristretta delle sue élites politiche, non è riuscito a mettere insieme, in sintonia e in relazione le masse alla nazione e così, fallendo, ha prodotto quella frattura tra Stato, masse e nazione, in cui consiste la debolezza intrinseca dello stato liberale europeo.

Ora, da questo punto di vista il fascismo non può che essere l'esito di questa trasformazione perché riuscì a realizzare quel progetto di nazionalizzazione delle masse, su cui aveva fallito lo stato liberale.

Nella misura in cui il fascismo realizzò l'integrazione tra popolo e nazione e intorno alla nuova idea di nazione, imperiale e totalitaria, plasmò l'identità del popolo italiano, per Volpe il fascismo poteva essere considerato l'erede del Risorgimento. Erede, ma anche sintesi superiore, capace di portare a soluzione i problemi che lo stato liberale aveva lasciato irrisolti.

Questo è il campo di questioni sulle quali Volpe ha lasciato la più significativa eredità storiografica, perché, rispetto alla storiografia antifascista che aveva attribuito il successo del fascismo quasi esclusivamente all'uso spregiudicato della forza e all'appoggio delle vecchie elites economiche, metteva in evidenza il suo più efficace punto di forza: la vittoria del fascismo risiedeva nell'aver saputo affrontare - e per Volpe risolvere - il rapporto tra masse e stato, tra classi lavoratrici e potere politico, che lo stato liberale aveva disatteso e che la Grande guerra aveva fatto esplodere.

\footnotetext{
${ }^{4}$ Benedetto Croce, Storia d'Italia dal 1871 al 1915, Bari, Laterza, 1928.

${ }_{5}^{5}$ Benedetto Croce, Storia d'Europa nel secolo decimonono, Bari, Laterza, 1932.

${ }^{6}$ Benedetto Croce, Per la nuova vita dell'Italia. Scritti e discorsi (1943 - 1944), Napoli, Ricciardi 1944 , pp. $55-56$.
} 
Lo strumento di cui il fascismo si avvalse per realizzare questo ambizioso progetto fu il corporativismo, presentato come alternativa al bolscevismo, ma anche la liberismo; eguaglianza e emancipazione sociale da un lato e dall'altro l'individualismo economico - che costituivano il baricentro ideologico delle due grandi ideologie dell'Ottocento venivano negati, ma anche sussunti all'interno del progetto corporativo, che metteva sotto il controllo dello stato la concorrenza e il conflitto sociale e valorizzava impresa e classe operaia come parti integranti del "lavoro nazionale".

In quest'ottica la nazione del fascismo è la nazione corporativa perché in esso poggia sia la possibilità materiale di fare dell'Italia una grande potenza mediterranea, sia la costruzione del mito totalitario.

Nel 1939, Volpe riversò questo modello interpretativo elaborato per spiegare le origini del fascismo in una sintesi della storia del regime che apparve con il titolo di Storia del movimento fascista, ${ }^{7}$.

Quando Volpe mise a punto questo suo nuovo libro evano passati più di dieci anni dal suo lavoro precedente nei quali il fascismo aveva non solo consolidato il suo potere in Italia, ma soprattutto si era allargato in molti paesi europei, non solo la Germania di Hitler, ma anche nello Estado novo di Salazar, e infine nella Spagna franchista. La trama interpretativa risultava una conferma del modello interpretativo elaborato ne L'Italia in cammino: una grande forza non solo italiana ma europea che attraverso il mito della nazione aveva rigenerato e unificato il vecchio continente.

Questo risultatato appariva la più indiscutibile smentita alla visione di Croce, che aveva presentato in fascismo come l' "invasione degli Icxos", un'irruzione brutale dell'irrazionalità, destinata a esaurirsi perché estranea alle correnti fondamentali dell'evoluzione progressiva della società europea. Per Volpe invece il fascismo non era un "accidente e un incidente» della storia italiana ${ }^{8}$, bensì un moto profondamente autentico e originale della storia nazionale, che, inoltre, aveva dimostrato di avere un notevole forza propulsiva su scala continentale e che alla vigilia della seconda guerra mondiale ambiva a proiettarsi in una dimensione mondiale.

Nello stesso anno, il secondo studioso del quale intendo occuparmi, Francesco Ercole, pubblicava la sua Storia del Fascismo. In quell'anno Ercole stava concludendo la sua esperienza di deputato,era professore di storia all'Università di Roma e presidente dell'Istituto storico italiano per l'età moderna, che nel 1935 aveva fondato lo stesso Gioacchino Volpe. Il suo modello storiografico però si distanzia notevolmente da quello di Volpe, perché pone l'accento più sulla discontinuità che sulla continuità del fascismo con il passato della storia risorgimentale.

Nell' impianto di Gioacchino Volpe il fascismo è in continuità con il Risorgimento anche se la sua affermazione è avvenuta attraverso una rottura istituzionale che possiamo persino chiamare rivoluzione, ma l'elemento centrale di questa concezione è la continuità dello stato nazionale che trova nel fascismo la sua massima espressione. Ercole, invece, appartiene ad un'altra scuola di pensiero, che intende ed interpreta il fascismo come rivoluzione, cioè come un movimento di rottura con lo stato liberale, che introduce nella storia d'Italia un elemento di discontinuità rivoluzionaria.

\footnotetext{
${ }^{7}$ Gioacchino Volpe, Storia del movimento fascista Milano, Istituto per gli studi di politica internazionale, 1939.

${ }^{8}$ Enzo Collotti, Fascismo, fascismi, Firenze, Sansoni, 1989, p. 11.
} 
Si tratta di una lettura di straordinario interesse per gli studi storici successivi, perché Ercole, che né ai suoi tempi, né successivamente venne considerato un grande storico tanto da finire nel più totale oblio insieme alle sue opere, pone con questo suo lavoro una serie di questioni di grande rilievo.

In prima istanza affronta il fascismo su scala europea, fin dalle sue origini, che stanno più nelle fratture della Grande guerra che nei problemi irrisolti del Risorgimento. Nel crogiolo della guerra mondiale emergono tre soluzioni al problema che essa stessa ha aperto, e cioè la necessità di integrare masse notevoli di lavoratori e di piccola borghesia all'interno dello stato. Una prima soluzione è quella proposta dal comunismo, che è concepito più che come avversario del fascismo, come movimento competitore che con il movimento fondato da Mussolini condivide i caratteri di novità e di rottura; l'altra soluzione è il progetto democratico, cioè la trasformazione degli stati dell'Europa occidentale e degli Stati Uniti da liberali a democratici. Il fascismo si inserisce in questo snodo, proponendosi come la "terza via", nella quale i processi di democratizzazione e di massificazione della società ("Noi siamo la vera democrazia", dirà Mussolini) vengono sottoposti al controllo di uno stato forte, autoritario e onnipresente, che si propone non solo come sintesi tra popolo e nazione, ma anche come supremo organizzatore della produzione attraverso il corporativismo.

Per Ercole, dunque, l'idea "fascista" è profondamente rivoluzionaria, e contende al comunismo bolscevico il primato dell'innovazione politica e della rottura con il passato liberale ottocentesco. Negli anni venti sono in marcia due rivoluzioni e l'uscita dal liberalismo è possibile attraverso un'alternativa: la rivoluzione comunista e la rivoluzione fascista che, lungi dall'essere una controrivoluzione o una mera reazione al comunismo, propone all'Europa una strada nuova e originale per la riorganizzazione dei sistemi politici dopo la "grande trasformazione" della guerra mondiale.

Alla luce di quello che stava accadendo alla fine degli anni Trenta - che è il punto di osservazione del nostro storico - era possibile ipotizzare che nel confronto delle due "vie" rivoluzionarie, quella vincente e dotata di maggior forza propulsiva fosse proprio il fascismo. Infatti alla vigilia della seconda guerra mondiale la "terza via" declinata in diversi esperimenti semi o para fascisti, più o meno lontani dal modello totalitario originale, sembrava decisamente aver avuto la meglio quantomeno in termini di consenso e di forza politica.

L' operazione storiografica e ideologica di Ercole mirava all'interpretazione del XX secolo nel suo complesso, che muoveva dalla considerazione secondo cui il dopoguerra era stato un periodo caratterizzato dalla conclusione del ciclo liberaldemocratico, iniziato nell'Ottocento, e dall'inizio di una nuova epoca dominata, per lo meno nell'Europa centro-occidentale, dal fascismo. Il fascismo, dunque, non è affatto un movimento nazionale e circoscrivibile al solo caso italiano, ma è un movimento europeo, in virtù della sua duttibilità ad essere declinato in tanti modelli, ma che mantiene la sua unitarietà grazie alla forza dello stato corporativo.

In ciò consiste la soluzione all'antitesi tra il totalitarismo comunista e l'anarchia "democratica" degli stati borghesi. La crisi degli anni Trenta dette ovviamente molta energia alla teoria corporativista che apparve come uno strumento molto efficace per lo sviluppo delle identità nazionali e per l'integrazione tra popolo e masse, in un epoca di straordinarie turbolenze economiche e di forti contrasti sociali. A dimostrarlo sta il fatto che anche Roosevelt e gli uomini del New Deal guardavano al corporativismo 
come ad un'esperienza storica di grande attualità e da cui attingere per uscire dalla crisi spaventosa in cui gli Stati Uniti erano rovinosamente precipitati; un atteggiamento destinato a non rimanere isolato al solo caso d' oltreoceano, ma che coinvolse anche le socialdemocrazie europee impegnate in una riflessione sull'economia pianificata.

A partire da queste considerazioni Ercole propone un'altra riflessione, che si allaccia strettamente a molte discussioni emerse anche in queste giornate di studio; la constatazione cioè che dove c'è il corporativismo, c'è il totalitarismo. Detto in altri termini: laddove si impianta un modello sociale ed economico di tipo corporativo lì emerge e si rafforza la deriva totalitaria, ossia una nuova forma di organizzazione statuale nella quale ogni aspetto della vita civile è posto sotto il controllo del partitostato e si realizza una compiuta integrazione tra stato e società.

Una riflessione che Ercoli propone per una ragione molto semplice e precisa: dimostrare un primato dell'Italia rispetto alla Germania, come paese precorritore e all'avanguardia, perché mentre quest'ultima, la Germania, ha fatto fatica a costruire un suo stato totalitario, l'Italia è stata la prima a realizzarlo e costituisce il modello sulla base del quale le altre nazioni plasmeranno il proprio stato corporativo. Questa nuova forma/stato non si esaurisce in una semplice ingegneria istituzionale, ma costituisce il centro di irradiazione di un progetto politico destinato a essere tanto più totalitario, quanto più la soluzione corporativa si rafforzerà e si dispiegherà in tutte le sue implicazioni.

Certo Mussolini in più di una occasione ha sostenuto - e questo dovrebbe insegnare qualcosa a noi storici che spesso discutiamo più di nominalismi che di sostanza - che per realizzare il totalitarismo fascista ci sarebbe voluto un secolo; il destino totalitario dell'Europa era dunque un processo in costruzione, un cammino in corso anche nell'Italia, che era stata la prima a incamminarsi su quella via. Per il Duce la generazione degli uomini che aveva scoperto nelle trincee della Grande guerra la "seduzione totalitaria", aveva solo compiuto il primo passo; certamente quello più difficile e rivoluzionario, ma l'effettiva costruzione dell'Europa totalitaria sarebbe stato un compito delle generazioni a venire.

In conclusione, con questo intervento ho inteso suggerire a noi storici contemporanei , che sarebbe un grave errore di prospettiva considerare la riflessione tra gli storici fascisti degli anni Trenta un'operazione puramente strumentale ed esclusivamente finalizzata all'esaltazione agiografica del regime. Trattare questi storici come meri propagandisti di partito, fa perdere di vista le opportunità che le loro riflessioni offrono all'interpretazione del passato, anche perché da questo imponente scavo storiografico, di cui ho dato in questa sede solo un piccolo scampolo, è derivata la cultura storica sulla quale si sono formate, attraverso i manuali scolastici, due generazioni di italiani. Nel 1935-1936, infatti, quando il ministro De Vecchi vira i programmi di insegnamento in direzione di un ulteriore rafforzamento del mito del regime, questo corpus interpretativo sul fascismo fornito dalla storiografia accademica si trasferirà senza molte mediazioni nei programmi d'insegnamento. Ma nel processo di trasferimento fu la proposta interpretativa di Gioacchino Volpe ad avere la meglio e la teoria della "continuità" prevalse sulla visione del fascismo come rivoluzione. 